\title{
Coronavírus e o comércio exterior: Estudo bibliográfico
}

\author{
Coronaviruses and foreign trade: Bibliographical study \\ Coronavirus y comercio exterior: Estudio bibliográfico
}

Recebido: 09/06/2021 | Revisado: 17/06/2021 | Aceito: 21/06/2021 | Publicado: 23/06/2021

\author{
Natalie de Fátima Alves Sebok \\ ORCID: https://orcid.org/0000-0001-5177-619x \\ Universidade Católica de Pernambuco, Brasil \\ E-mail: naty.sebok@gmail.com
}

\begin{abstract}
Resumo
Objetivo: analisar as informações sobre como o coronavírus surge como uma das grandes incertezas no cenário econômico em 2020. O impacto na economia mundial e quais são as novas projeções para os próximos meses. Os fatores e os riscos percebidos para a globalização, assim como os cenários para a evolução do comércio a nível mundial como essa crise mundial que vivenciamos. Metodologia: Trata-se de um estudo bibliográfico quantitativo feito a partir de uma triagem. A análise e reunião das informações foram baseadas em artigos já publicados sobre o coronavírus na cena mundial até o presente momento. Foi adaptado e compilado tudo que podemos mostrar como necessário ao entendimento sobre a situação atual sobre o comércio exterior. Conclusão: Percebeu-se com esse estudo bibliográfico o impacto causado no comércio exterior advindo de uma situação de saúde sanitária na qual não vivenciamos nada parecido nos últimos 100 anos. Quais as nossas expectativas e projeções esperadas para o próximo ano e quais foram as perdas até o presente momento.
\end{abstract}

Palavras-chave: Coronavírus; Impacto econômico; Cenários; Projeções; Covid-19.

\begin{abstract}
Objective: analyze information on how the coronavirus emerges as one of the great uncertainties in the economic scenario in 2020. The impacto on the world economy and what are the new projections for the coming months. The factors and risks perceived by globalization, as well as the scenarios for the evolution of trade worldwide, such as this global crisis that we are currently experiencing. Methodology: This is a bibliographic study, the analysis and gathering of information was based on articles already published about the coronavirus on the world scene to date. Everything we have shown has been adapted and compiled as necessary to understand the current situation regarding foreign trade. Conclusion: With this bibliographic study, the impact on foreign trade resulting from a health situation in which we have not experienced anything similar in the last 100 years was perceived. What are our expectations and projections expected for the next year and what were the losses to date time.
\end{abstract}

Keywords: Coronavirus; Economic impact; Scenarios; Projections; Covid-19.

\section{Resumen}

Objetivo: analizar información sobre cómo emerge el coronavirus como una de las grandes incertidumbres en el escenario económico en 2020. El impacto en la economía mundial y cuáles son las nuevas proyecciones para los próximos meses. Los factores y riesgos percibidos por la globalización, así como los escenarios de evolución del comercio a nivel mundial, como esta crisis global que vivimos actualmente. Metodología: Se trata de un estudio bibliográfico basado en artículos ya publicados sobre el coronavirus en el panorama mundial hasta la fecha. Todo lo que hemos mostrado ha sido adaptado y recopilado según sea necesario para comprender la situación actual en materia de comercio exterior. Conclusión: Con este estudio bibliográfico se percibió el impacto en el comercio exterior derivado de una situación de salud sanitaria en la que no hemos experimentado nada similar en los últimos 100 años. Cuales son nuestras expectativas y proyecciones esperadas para el próximo año y cuáles han sido las pérdidas hasta la fecha de hoy. Palabras Clave: Coronavirus; Impacto económico; Escenarios; Proyecciones; Covid-19.

\section{Introdução}

O mundo está padecendo de um novo vírus jamais observado, a Covid-19. A Fiocruz, assim como outras organizações, associa esse vírus aos morcegos, onde sofrem mutações e passam a infectar humanos. O primeiro caso surgiu segundo a Organização Mundial da Saúde (OMS) em 31 de Dezembro de 2019, que foi alertado sobre casos de pneumonia na cidade de Wuhan na China (Organização Pan-Americana da Saúde, 2020). 
Este artigo fará uma avaliação sobre os impactos que o coronavírus fez na economia mundial, junto ao comércio internacional e também sobre a política comercial. Considerando um cenário desconhecido onde não temos uma noção do que está por vir e muito menos o controle em sua grande parte, muitas análises tornam-se especulativas com o cenário que vivemos.

Teremos uma pesquisa a curto prazo, colocando diversas situações que foram vivenciadas nos anos de 2019 e comparadas ao ano de 2020 e como esses números influenciaram a política comercial e de investimentos. O objetivo é examinar a repercussão da COVID-19 no ano de 2020 dentro do comércio exterior e entender quais são as vantagens e desvantagens que essa crise sanitária nos ocasionou.

Analisando as informações sobre como esse vírus surge como uma das grandes incertezas no cenário econômico em 2020 os fatores e os riscos percebidos para a globalização nesse período, assim como os cenários para a evolução do comércio a nível mundial como essa crise sanitária que vivenciamos.

\section{Metodologia}

Trata-se de um estudo de estratégia de investigação qualitativa, pois com essa seleção metodológica conseguimos compreender um determinado problema social não só no âmbito de sua manifestação mas também diferenciar suas ressalvas (Ludke, \& Andre, (2013)).

Com essa metodologia obtivemos um entendimento através da triagem de diversos artigos publicados sobre como a Covid-19 atingiu o comércio exterior. Foi adotado como critério de inclusão: as informações feitas com base nas pesquisas referente ao período de 2020 sobre a situação sanitária existente no mundo juntamente com as ocorrências no comércio exterior no mesmo período.

As informações obtidas estão sendo passadas através de escrita e figuras apresentadas como tabelas e gráficos que foram publicados nos anos de 2020 e 2021. A análise interpretativa foi efetivada através de uma base crítica com o suporte dos textos analisados (Severino, 2007, p. 59).

\section{Resultados e Discussão}

\subsection{Comércio Mundial}

São tempos de muitas incertezas e incapacidade de exatidão dos próximos cenários político e econômico até que exista um controle mundial sobre a crise sanitária vivenciada atualmente. Observamos uma colisão e uma interrupção abrupta do ciclo comercial quando falamos de impactos sofridos com a logística do comércio exterior. Em logística humanitária o conceito de logística é associado ao estudo de como o gerenciamento pode proporcionar um melhor nível de lucratividade nos serviços de distribuição para clientes e consumidores, por meio de planejamento, organização e controle eficazes para atividade de manuseio e armazenamento, visando facilitar o fluxo de produtos (Ballou, 2006). As consequências para o comércio não será somente a nível tático e sim estratégico também. Relacionado a tudo que vivemos nos últimos cem anos, ocorreu a Grande depressão de 1930, a crise financeira de 2008-2009 entre outras crises como a Segunda Guerra Mundial, porém não conseguimos equiparar e muito menos comparar a atual crise presente no mundo. O desempenho do PIB é tradicionalmente o principal indicador que explica e condiciona a evolução dos fluxos de comércio internacional, ao menos na ausência de elementos que o restrinjam diretamente, como o aumento de tarifas e barreiras não tarifárias (IPEA, Carta de Conjuntura, 47, $2^{\circ}$ Trimestre de 2020, p. 4).

Com menos de 1 mês do vírus, a projeção do PIB brasileiro já apresenta variação oficial em torno de $2 \%$ para menos ( $2 \%$ para $0 \%$, e para alguns economistas de quase $4 \%$ para menos ( $2 \%$ para $-2 \%$ ).

O PIB brasileiro em 2019 foi de R \$7,3 trilhões. A expectativa do crescimento do PIB em 2020 no Brasil antes de existir a COVID-19 era de 2\%, porém já em março quando iniciou o nosso processo de lockdown no Brasil o PIB já foi calculado 
para 0\%. Portanto, com essa nova projeção o Brasil deixou de ganhar em torno de R \$150 bilhões. Podemos lembrar que o Brasil possui 2,3\% do PIB mundial e com a covid-19 termos um dano mundial em torno de 10 vezes a média anual de desastres (esta média anual está em torno de US \$153 bilhões).

Nesse gráfico observamos que no primeiro semestre existe uma queda elevada no PIB se confrontarmos com o segundo semestre onde procuramos voltar à regularidade em algumas atividades econômicas. Segundo o JP Morgan Global PMI (Purchasing Managers Index), a queda da atividade em março $(39,4)$ foi de $6,7 \%$ comparado a fevereiro $(46,1)$. "Para comparação, no pior momento da crise de 2009, o PMI ficou próximo de 37. O gráfico mostra que há uma elevada correlação entre o PMI e o PIB global”. (IPEA, Carta de Conjuntura, 47, n $2^{\circ}$ Trimestre de 2020, p. 4).

Figura 1. Correlação entre o PMI x GDP.

\section{Global PMI and GDP}

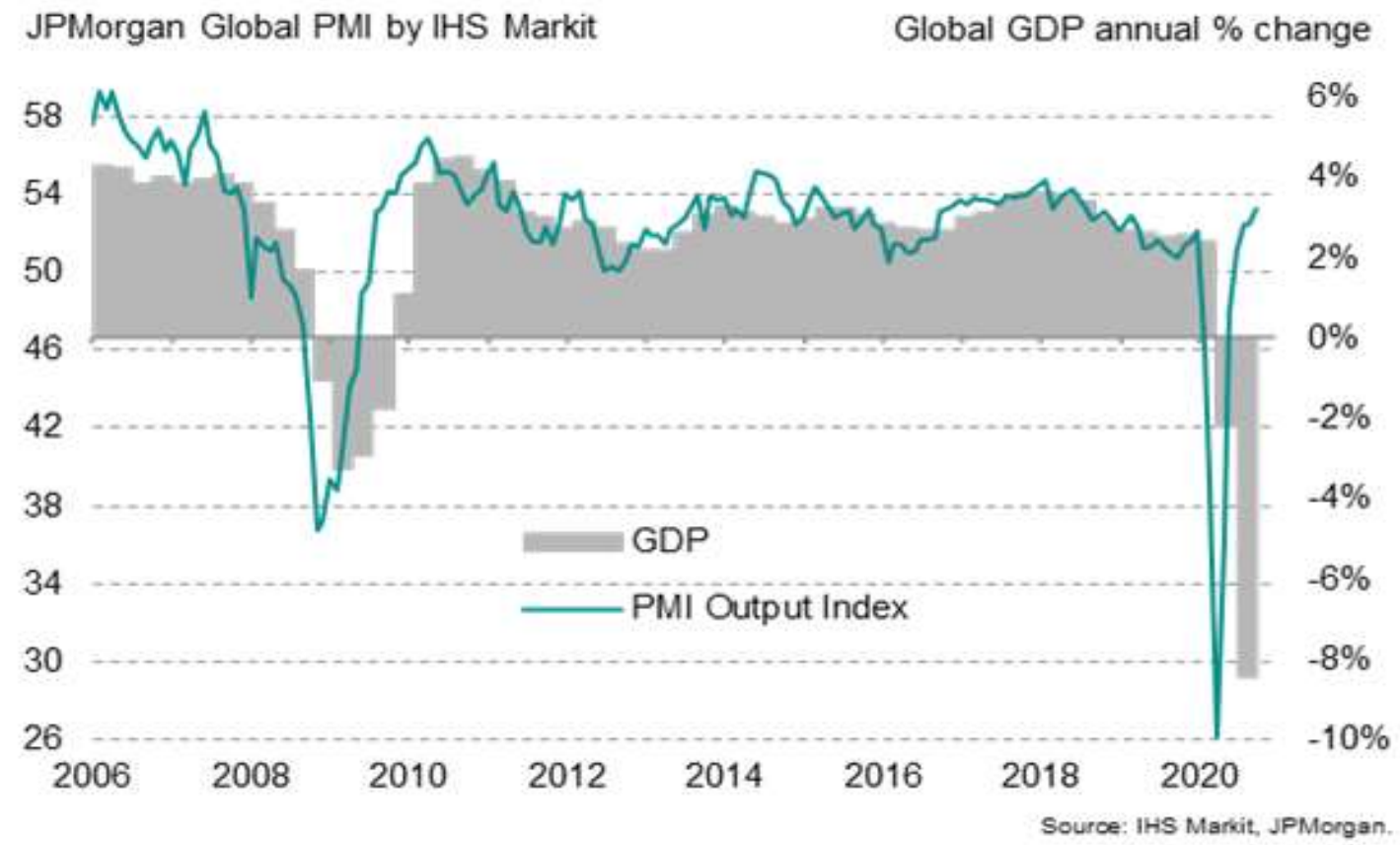

Fonte: IHS Markir, JPMorgan (2020).

Podemos observar também que de acordo com a pesquisa realizada pelo Jornal da USP feita pela Rede de Pesquisa Solidária, conseguimos analisar a intensidade do impacto causado pela pandemia no comércio internacional brasileiro comparando as importações e exportações dos primeiros seis meses de 2020 com o mesmo período nos meses de 2019. Nesse gráfico vemos um declínio de $20 \%$ no mês de janeiro de 2020, contraposto ao mesmo período de 2019. Em fevereiro essa comparação foi reduzida e em março a exportação cresceu 5,4\% comparado a 2019. Nos meses de abril, maio e junho as exportações voltaram a ficar negativas. 
Figura 2. Variação percentual das exportações 2019/2020.

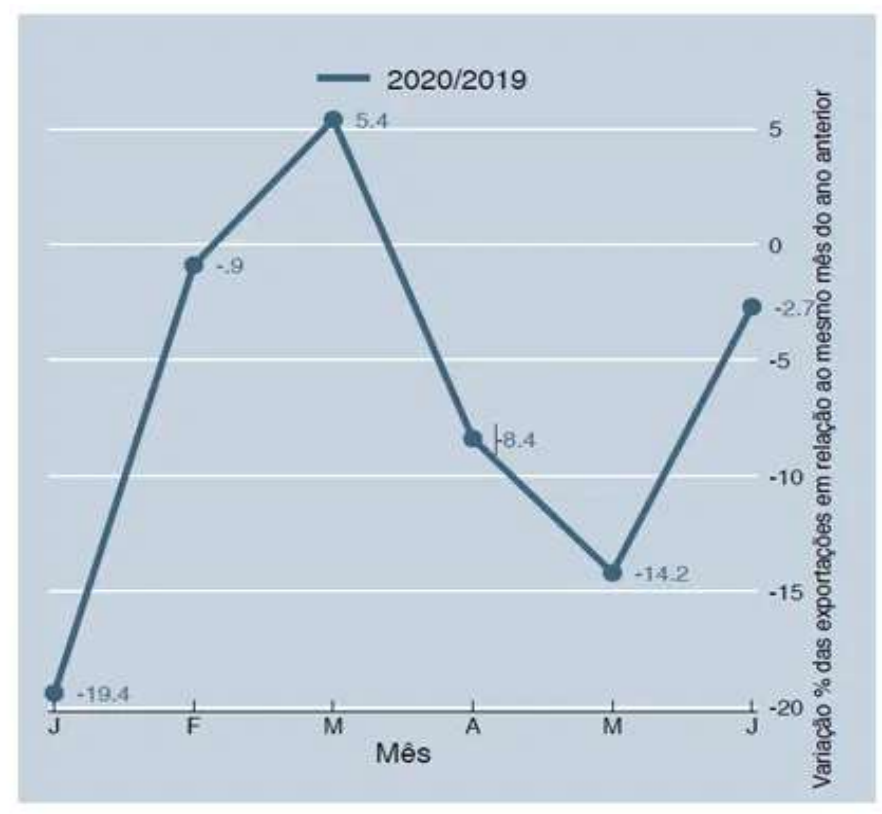

Fonte do Gráfico: Jornal da USP. (11/08/2020)

Pela Organização para a Cooperação e Desenvolvimento Econômico (OCDE) o impacto sobre o PIB de diversos países tomando como referencial a oferta e demanda, os setores que mais foram prejudicados são os que atuam na área de serviço e entretenimento juntamente com a indústria e a construção. Nesta pesquisa observamos uma queda de 50\% e $100 \%$, e o impacto no PIB seria em torno de $15 \%$ a $30 \%$, pois estes dependem das pessoas em uma forma presencial para acontecerem. Observando os países onde a agricultura tem um peso grande relacionado ao PIB, podemos atentar uma repercussão menor na queda dos faturamentos anuais. Em contrapartida quando falamos de serviços essenciais como alimentos (ao contrário do contemplamos nos outros setores falados anteriormente) não teremos um impacto neste setor, muito pelo contrário o mesmo terá um aumento.

O faturamento da indústria de alimentos segundo a Agência Brasil.ebc foi de R $\$ 789,2$ bilhões em 2020, que se confrontarmos com 2019 o resultado foi de $+12,8 \%$. Esta quantia chega aos 10,5\% do PIB nacional, e esses dados são referentes a Associação Brasileira da Indústria de Alimentos (ABIA). Este resultado foi validado a partir do aumento das vendas de varejo em $16,2 \%$ e para o mercado externo de $11,4 \%$. Os produtos que mais ganharam com a pandemia foram os açúcares com uma elevação de 58,6\%, os óleos vegetais com 21,2\% e as carnes com uma aumento de $13 \%$ comparados ao mesmo período de 2019.

Apesar de vivermos uma crise sanitária, a indústria alimentícia nas exportações em 2020 teve um acréscimo de 11,4\% comparado a 2019, rematando um total de US\$38,2 bilhões. Esses números resultaram em uma atuação de $25 \%$ nas vendas do setor, o que ocasionou um aumento conferido a 2019 (19,2\%) de 5,8\%.

"Atuando com agilidade e adotando com rigor todos os protocolos de segurança, o setor conseguiu aumentar sua produção e não deixou faltar comida à mesa dos brasileiros” destacou o presidente executivo da ABIA, João Dornella.

Segundo a ABIA, como o câmbio brasileiro sofreu uma excessiva desvalorização e juntamente com isso uma forte demanda de importação pela Ásia principalmente pela China, podemos obter bons números nos nossos resultados finais apesar de estarmos com todo o quadro de crise financeira mundial vivida pela COVID-19. 
Figura 3. Exportações brasileiras do agronegócio - Participação da China (2020 - Jan/Jul).

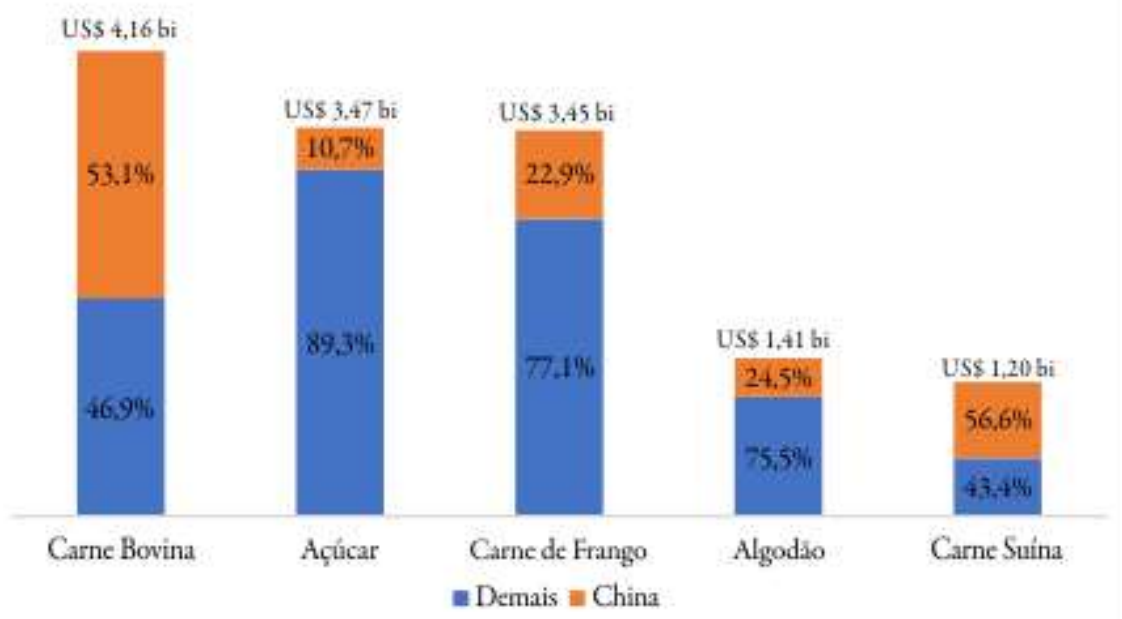

Fonte: AgroStat Brasil a partir dos dados da Secex/ME. Elaboração: Mapa/SCRI/DNAC/CGEA. Dados extraídos em agosto de 2020. Sujeitos à alteração. Notas: 1. Carnes in natura. 2. Açúcar de cana em bruto. 3. Algodão não cardado e não penteado.

Atentamos com esse gráfico que a China foi o nosso principal importador, e no primeiro semestre a demanda chinesa consumiu $40 \%$ do total do valor exportado em 2020. As exportações para todo o mundo, entre janeiro e julho de 2020, foram de US \$ 4,16 bilhões para carne bovina in natura (+32,3\%), US\$3,45 bilhões para carne de frango in natura $(-11,87 \%)$ e US\$1,20 bilhão para carne suína in natura (+51,70\%; recorde em valores e quantidade) segundo a FUNCEX.

Não obstante o que aconteceu no ano de 2020, em 2021 poderemos considerar um início de uma normatização para o que chamamos de cenário favorável ao PIB (um crescimento esperado), já que devemos fechar nosso ano de 2020 com uma queda de $-0,5 \%$ a $-3,5 \%$. Com esse valores podemos desenhar alguns cenários para a evolução: um crescimento próximo ao que seria hoje o crescimento potencial da economia mundial, de cerca de 3,5\%; uma forte recuperação após a recessão, a exemplo do que ocorreu em 2010, com crescimento da ordem de 5\%; e uma retomada lenta da atividade, com parte dos efeitos negativos da crise atual se estendendo aos primeiros meses do próximo ano, o que limitaria o crescimento a cerca de $2 \%$. Para transpor esses cenários de crescimento do PIB para cenários de crescimento do comércio mundial, seria preciso aplicar uma elasticidade. Estimativas da elasticidade de longo prazo entre importações e PIB mundiais encontram valores entre 2 e 3 , dependendo do método utilizado e do período analisado (Constantinescu, Mattoo, Ruta \& Hoekman, 2015).

A partir do que vimos, podemos analisar que cada ponto percentual que cai em relação ao PIB mundial, representa vários pontos que perdemos no comércio, podemos dizer que em torno de $25 \%$ será a nossa queda comercial caso tenhamos uma redução de $3,5 \%$ no ano de 2020. Porém com a melhora do cenário mundial relacionado a covid e tudo que ela nos traz como a paralisação do comércio já citada, podemos dizer que teremos uma recuperação do PIB em 2021. 
Figura 4. Variação das Importações Mundiais em 2020/2021.

\begin{tabular}{|c|c|c|c|c|c|}
\hline 2020 & 2021 & \multicolumn{4}{|c|}{ Acumulado 2020-2021 } \\
\hline \multirow{3}{*}{$-20,0$} & 4,0 & $-16,8$ & 0,8 & 1,04 & $(16,8)$ \\
\hline & 7,0 & $-14,4$ & 0,8 & 1,07 & $(14,4)$ \\
\hline & 10,0 & $-12,0$ & 0,8 & 1,1 & $(12,0)$ \\
\hline \multirow{3}{*}{$-15,0$} & 4,0 & $-11,6$ & 0,85 & 1,04 & $(11,6)$ \\
\hline & 7,0 & $-9,1$ & 0,85 & 1,07 & $(9,1)$ \\
\hline & 10,0 & $-6,5$ & 0,85 & 1,1 & $(6,5)$ \\
\hline \multirow{3}{*}{$-25,0$} & 4,0 & $-22,0$ & 0,75 & 1,04 & $(22,0)$ \\
\hline & 7,0 & $-19,8$ & 0,75 & 1,07 & $(19,8)$ \\
\hline & 10,0 & $-17,5$ & 0,75 & 1,1 & $(17,5)$ \\
\hline
\end{tabular}

Fonte: IPEA, Carta de Conjuntura, 47- 2º Trimestre de 2020, p. 11.

Pode-se imaginar um cenário otimista no qual haverá uma recuperação comercial considerável em 2021, porém temos que levar em conta as barreiras não tarifárias e os conflitos comerciais que irão aumentar nas potências mundiais como China e Estados Unidos.

\section{Conclusão}

Os estudos sobre o novo vírus e a concentração de esforços contra o tempo para que exista uma forma de imunização mundial a essa crise sanitária, levou a comunidade científica a desenvolver o mais rápido possível a vacina eficiente para que a sociedade volte a regularização das atividades econômicas e sociais. Não obstante a sociedade mudou e provavelmente evoluiu também para um novo comércio digital. Esse comércio também influenciou o PIB mundial já que a grande parte dos serviços e produtos ofertados pelo meio digital também são fundamentais para a sobrevivência das pessoas (Marketing digital, E-commerce e pandemia: uma revisão bibliográfica sobre o panorama brasileiro. (2020). p.5).

Com esse estudo, pude buscar bibliografias e fazer uma avaliação sobre os impactos que o coronavírus vêm trazendo para o comércio mundial. Fazendo considerações, comparações e análises quantitativas dos cenários apresentados em 2019 e 2020 e apresentar uma suposta avaliação para 2021. Foi efetuado a análise em cima das projeções de queda do PIB por conta dos impactos que a pandemia acarretou. Levando em consideração a variação do PIB que poderá ser de -0,5\% a -3,5\% em 2020, teremos uma queda de mais ou menos $20 \%$ no comércio no cenário pessimista, em contrapartida vimos que o setor de alimentação cresceu apesar da covid-19.

O aparecimento dessa crise sanitária, eclodiu em um momento delicado para as relações comerciais mundialmente falando. Podemos articular também que passando esta crise, a economia mundial terá uma crescente de negócios onde iremos recuperar o nosso PIB perdido em 2020 através de negócios multilaterais.

A Covid-19 nos trouxe também consequências para o investimento estrangeiro direto pela queda do comércio deste, trazendo um aprendizado de que em situações de falta de matéria prima, podemos fazer uma cadeia de fornecimento diferente da atual, assim promovendo uma abertura comercial maior. Embora tal iniciativa esteja fora das prioridades de curto prazo em função da pandemia, é importante que o país sinalize o compromisso com a abertura no futuro. Finalmente como qualquer crise vivida há cem anos ou nos dias atuais, esses momentos apresentam enormes barreiras e desafios, nos quais podemos criar mecanismos e oportunidades para atingir os objetivos, as estratégias e as políticas mais adequadas a cada situação que nos ocorre. Podemos dizer que com uma situação na qual o mundo se encontra hoje, o mais recomendável é que sejam feitas políticas e estratégias para que se consiga sair desta situação com a menor taxa de perdas possíveis, e que assim a reversão do quadro seja breve para que os ganhos nas questões financeiras avancem novamente, sempre investindo em ciências, inovações e tecnologias. 
Frisamos que ocorre insuficiência de dados sobre o caso da covid-19, já que nos dias atuais o vírus ainda está mundialmente afetando a economia e a sociedade. Por consequência disso existem limitações de informações que só serão concluídas e estimadas quando a pandemia estiver controlada ou até mesmo acabada, que será o momento em que conseguiremos ter acesso ao real impacto global que ela causou. Por conta disto este estudo foi baseado em dados e efetivado através de uma seleção de informações existentes porém limitadas, na qual acreditamos que venha a colaborar com estudos necessários sobre a questão no futuro, onde conseguiremos analisar com clareza o que teremos que fazer para que toda a nossa economia volte à normalidade esperada.

Só será possível analisar as repercussões que o coronavírus trouxe ao PIB mundial no futuro e com isso tomar as decisões necessárias para que o mundo volte a percorrer seus caminhos de ganhos econômicos contrapondo o período pré, durante e o pós coronavírus.

\section{Referências}

AgroStat Brasil a partir dos dados da Secex/ME. (2020). Elaboração: Mapa/SCRI/DNAC/CGEA.

Aslam, A. E. (2017). et al. Global trade: drivers behind the slowdown. Voxeu.org.

Auboin, M. \& Borino, F. (2017). The falling elasticity of global trade to economic activity: testing the demand channel. WTO.

Ballou, R. H. (2006). Gerenciamento da cadeia de suprimentos/Logística Empresarial. Editora Bookman,

Bloningen, B. A. \& Prusa, J. (2015). Dumping and antidumping duties. Massachusetts: NBER. Working Paper, n 21573.

Bocchini, B. (2020). Faturamento da indústria de alimentos cresce 12,8\% em 2020. https://agenciabrasil.ebc.com.br/economia/noticia/2021-02/faturamentoda-industria-de-alimentos-cresce-128-em-2020

Borin, A. (2017). et al. The cyclicality of the income elasticity of trade. Munich: MPRA. (Munich Personal RePEc, n. 77418).

Bussière, M. (2013). et al. Estimating trade elasticities: demand composition and the trade collapse of 2008-09. American Economic Journal: Macroeconomics, (5), 118-151.

Constantinescu, C., Mattoo, A. \& Ruta, M. (2015). The global trade slowdown: cyclical or structural? Washington: World Bank.Policy Research Working Paper, n. 7158.

Evaluating the initial impact of COVID-19 containment measures on economic activity - OECD. oecd-ilibrary.org

Fiocruz. (2020). O que é o novo coronavírus? https://portal.fiocruz.br/pergunta/o-que-e-o-novo-coronavirus

Hank, L. \& Fukunari, K. (2010). The internationalization of small and medium enterprises in regional and global value chains. Tokyo: ADBI. (ADBI Working Paper Series, n. 231).

Hoekman, B. (2015). (Ed.) The global trade slowdown: a new normal? London: CEPR. Vox EU E-book.

IHS Markit. (2020) Fonte do gráfico JP Morgan Global PMI. https://ihsmarkit.com/research-analysis/global-pmi-indicates-fastest-growth-for-over-twoyears-Nov20.html

Jones, R. \& Kierzkowski, H. (2005). International fragmentation and the new economic geography. North American Journal of Economics and Finance, v. 16, n. 1, p. 1-10.

Jornal da USP. (2020). Pandemia de Covid-19 reduz exportações brasileiras de bens de alta complexidade. https://jornal.usp.br/ciencias/pandemia-de-covid19-reduz-exportacoes-brasileiras-de-bens-de-alta-complexidade/

Ludke, M. \& André, M. E. D. A. (2013). Pesquisa em educação: abordagens qualitativas. E.P.U.

Oliveira, I., Honório, K., Ribeiro, F., Baumann, R., Oliveira, G. A., Giesteira, L. F., Tironi, L. F., Alves, A. P. (2020). Carta de Conjuntura, $n^{\circ} 47$ - $2^{\circ}$ Trimestre. https://www.ipea.gov.br/portal/index.php?option=com_content\&view=article\&id=35873\&Itemid=3

Machado, E. (2020). Coronavírus e o Comércio Exterior. https://www.jb.com.br/pais/artigo/2020/03/1022959-coronavirus-e-o-comercio-exterior.html Organização Pan-Americana da Saúde. (2020). Histórico da pandemia de Covid-19. https://www.paho.org/pt/covid19/historico-da-pandemia-covid-19

Ribeiro, O. L. \& Domingues, G. C. (2020). O desempenho das exportações do agronegócio nos primeiros sete meses de 2020. https://funcex.org.br/info/rbce145-outubro-dezembro-2020/o-desempenho-das-exportacoes-do-agronegocio-nos-primeiros-sete-meses-de-2020

Severino, A. J. (2007). Metodologia do trabalho científico. (23a ed.), Cortez

Silva, W. M., Morais, L. A., Frade, C. M. \& Pessoa, M. F. (2020). Marketing digital, E-commerce e pandemia: uma revisão bibliográfica sobre o panorama brasileiro. p.5. https://www.rsdjournal.org/index.php/rsd/article/view/15054/13586 